\title{
EFFECTIVENESS OF APPLICATION OF MODAL ANALYSIS FOR THE MONITORING OF STRESSED OR OPERATED STRUCTURES
}

\author{
Aleksey MIRONOV ${ }^{1}$, Pavel DORONKIN ${ }^{1}$, Aleksander PRIKLONSKY ${ }^{1}$, Andris CHATE ${ }^{2}$ \\ ${ }^{1}$ D un D centrs, Margrietas 7, LV-1046, Riga, Latvia \\ E-mail: aleksei@ddcentrs.lv \\ ${ }^{2}$ Institute of Materials and Structures, Riga Technical University, \\ Azenes 16-353, LV-1048, Riga, Latvia \\ E-mail: Andris.Cate@rtu.lv
}

Received 02 July 2015; accepted 14 September 2015

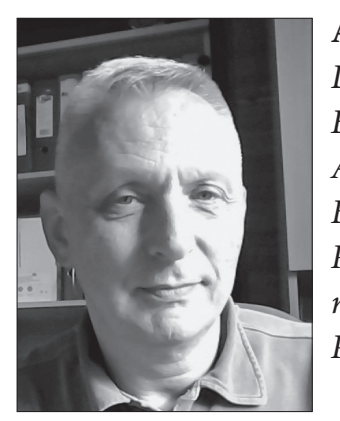

Aleksey MIRONOV, Doctor of Science (Engineering).

Date and place of birth: 1954, Tashkent, Uzbekistan.

Education: Riga Institute of Civil Aviation Engineering, Latvia.

Affiliations and functions: since 2004, scientific director of D un D centrs and Chairman of the Board of Aviation Research Centre since 2014.

Research interests: vibration diagnostics of turbine engines, bearings, gears, structural health monitoring of aviation and transport structures.

Publications: author of over 70 scientific papers.

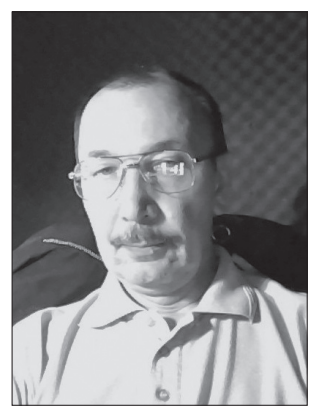

Pavel DORONKIN, Engineer.

Date and place of birth: 1956, Almaty, Kazakhstan.

Education: Riga Institute of Civil Aviation Engineering, Latvia.

Affiliations and functions: technical director of D un D centrs, board member of the

Vibroacoustic Laboratory since 2006.

Research interests: monitoring systems for aviation and civil structures applications, vibration and structural analysis, experimental testing equipment and software.

Publications: author of over 35 scientific papers.

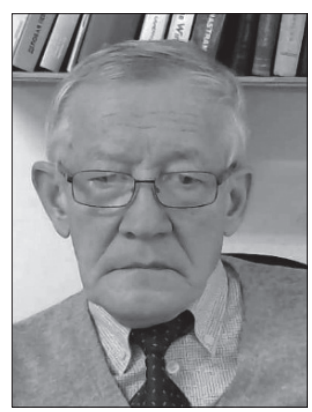

Alexander PRIKLONSKIY, Doctor of Science (Engineering)

Date and place of birth: 1948, Riga, Latvia.

Education: Riga Institute of Civil Aviation Engineering, Latvia.

Affiliations and functions: leading researcher of D un D centrs since 2008.

Research interests: vibration diagnostics of turbine engines, bearings, structural analysis and modelling of aviation and transport structures.

Publications: author of over 30 scientific papers.

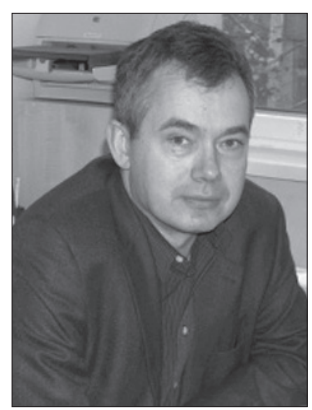

Andris CHATE, Doctor of Science (Engineering)

Date of birth: July 5, 1952, Cesis, Latvia.

Education: 1981 - PhD in mechanical engineering, Riga Technical University.

Affiliation and function: professor and head of the Institute of Materials and Structures, Riga

Technical University.

Fields of research: strength and dynamics of structures, damping analysis, mechanics of composite materials and structures, fracture mechanics.

Publications: author of over 150 scientific papers. 
Abstract. This paper presents a brief summary of the research results of the application of experimental and operational modal analysis (EMA and OMA) effectiveness for the assessment of the change in the condition of operating structures by modifying their dynamic characteristics. Special focus is given to the topicality of the operational modal analysis for the definition of the dynamic features of the structures (frequencies, modes and deformation) under near-natural conditions. The research was conducted using two operating laboratory models, when the first one imitated a part of a fuel/gas pipeline and the second - a helicopter blade. The results of finite-element model simulation, identification of natural mode and the influence of the two types of virtual defects on the changes in the dynamic properties are provided. The work describes the results of experimental research of the dynamic behaviour of the pipe model, using the methods of OMA and comparing them with the modelling results. The research results demonstrate how the modification of the condition of the large-scaled models impacts pipe and blade models when the defects of local and global nature are introduced.

Keywords: modal analysis of experimental pipeline and blade model, structural monitoring, diagnostic indicators.

\section{Introduction}

Structural Health Monitoring (SMH) as a way of scientific and technical activity has been formed in the last third of the $20^{\text {th }}$ century, uniting in itself data acquisition, computing, valuation and data analysis in order to control the technical condition of structures throughout their life cycle (Hall 1999). Simply stated, SHM defines the approach that provides the possibility to reveal dangerous changes of a construction resulting from caused damage or due to functionality (Kessler et al. 1978). Such "changes" or damage signature define the requirements to sensors that form the rest of the system components. The types of transportation, like aviation or pipe transport, have the highest potential return from prevention of the damages with the assistance of SHM, otherwise disasters are very expensive. For instance, to reduce risks, regular technical maintenance of airplanes is required, which ultimately results in as much as $27 \%$ of the cost of the aircraft life cycle (Hall, Conquest 1999).

Today, the main methodical tools of SHM are the methods of modal analysis. Experimental modal analysis is the process of defining the system modal parameters through an experimental method, which is lineal and independent from time (Allemang 1999). Initially, experimental analysis was used for refining the analytical model of the construction, and, if it is absent, for defining its main parameters. Later, experimental modal analysis started to be applied for solving the problems of dynamics, specifically, vibrations or acoustics, by analysing the system's behaviour as a reaction to excitation, described by modal parameters, combined with the boundary conditions. The modal analysis theory explains the existence of the Eigen frequency, deformation coefficient and the shape of natural modes for lineal systems that are the main modal parameters of the system. The most widespread practical application was found for lumped or discrete models, as well as for the distributed models, which view normal modes in terms of a possible solution for the modal parameters (Tse et al. 1978). The process of modal analysis traditionally includes 4 stages, including: modelling, data acquisition, valuation of modal parameters, and representation and validation of modal data. Modal analysis, as a rule, is based on the application of matrix differential equations, which take the finite-element mathematical form in consent with the measured data. This can be input data or output data in a temporary or frequent area (Bendat, Piersol 1980), processed, for instance, in the form of the impulse response or frequency response functions (IRF or FRF). The data acquisition includes all practical aspects of receiving the data, as well as measurement, digital processing of a signal and receiving data that are required as an input data at the modal parameter valuation stage (Dally et al. 1984). The valuation of modal parameters is connected with the choice of a mathematical model, based on the measured data and the record of systematic errors and, for instance, non-lineal distortions (Jolliffe 1986; Ljung 1987). The modal analysis is completed with the representation and validation of the modal data, including the physical interpretation of modal parameters.

The defect location strategy is based on the changes of dynamic behaviour of the structure, represented in the form of modal parameters (Magpantay 2006). For this purpose, it is necessary to evaluate the modal parameters of the object, which is not an easy task for active structures. In contrast with laboratory conditions, where evaluation of the modal parameters can be done with the help of test excitation, this method is rarely applicable for transport structures. Yet, at the same time, external disturbance influences these structures, as, for example, the air flowing over the element of the fuselage, or gas or liquid pumped through a pipe. This kind of influence can be sufficient for the excitation of natural modes, but difficult and most often quite impossible to measure.

Since the beginning of the 90's, Operational Modal Analysis (OMA), also referred to as the analysis of output data or of natural excitation, has attracted attention with regards to big civilian objects, and later aviation. This method only uses the response of the stressed structure excited by the surrounding environment for the valuation of modal features. For aviation and other modes of transport, the OMA application is attractive for several reasons: 1) low cost and ease of measuring, which do not require any advance preparation; 
2) the opportunity to receive the dynamic characteristics of the whole structure, not just its individual components; 3) model characteristics are linearised due to a wide range of accidental excitations; 4) all or some parts of the measured degree of freedom can be used for reference purposes, which significantly increases the method resolution and helps to segregate the paired and closely located modes; 5) the role of OMA is not limited to being a refining tool for structural improvements, it also proved to be useful for the vibration-based health monitoring (Zhang et al. 2005). Its widest application was found for the approaches using conversion in the time domain: the Natural Excitation Technique (NExT), the model of the general Auto-Regression Moving Average vector (ARMA V), the Stochastic Subspace-based methods, and two approaches in the frequency domain: the Frequency Domain Decomposition (FDD) and the leastsquares complex frequency-domain (LSCF).

This article examines the application of OMA methods for monitoring the status of stressed and operating structures subject to dynamic impact. Fixed structures like pipelines with an internal flow as well as moving structures, including rotating helicopter blades surrounded by circumfluent air flow, may be classified as dynamically stressed structures. For this purpose, the OMA has been applied increasingly since the beginning of the 2000's.

In (Lisowski et al. 2000) the Modal Analysis was applied to a helicopter for assessing and improving the ground-based test models, as well as for refining a FEM helicopter model so that it would hold out the flight conditions. Similar tasks were solved in (Basseville et al. 2001) for the booster of Ariane $V$ and a helicopter. The authors Kiddy, Pines (2001) came the closest to solving the problem of the on-line monitoring of a rotating blade. Their Modal Analysis method developed for a non-rotatable structure was used for detecting the ballistic defect of a blade. The method was applied on two kinds of structures: a beam rotating in a vacuum and a non-rotatable blade of the Hughes TH-55A helicopter. The method proved capable of bringing out the defects with a high modal power. At the same time, while detecting the defects with a low modal power, the authors faced a number of problems.

The task that the authors of the research set was to carry out an experimental test and to verify the applicability of the OMA for dynamically stressed objects under operating conditions that are close to natural. The efficiency criterion for this method was the degree of reliability in detecting the various scale test defects ranging from global defects that caused changes in the modal parameters of the whole object to local ones, the impact of which was limited to the damage area. Within the scope of the core task, the possibility of application of various types of SHM sensors - acceleration and deformation - was also considered.

\section{Main provisions}

The methods of Modal Analysis make it possible to experimentally determine the dynamic characteristics of a structure, including the frequencies, mode shapes and modal damping. Any structural defect related to the loss or redistribution of mass (erosion, chipping/flaking, deformation), rigidity (splits and cracks, changes of the physical and mechanical properties) and damping capacity (material ageing) causes changes in the condition of the structure, which means that any modification of the technical condition of a structure may be evaluated by its dynamic properties.

The methods of modal analysis that make use of the harmonic or shock test excitation $F(\omega)$ of the studied structures and measure the response $X(\omega)$ of these structures (Allemang 1999) became most widely applied when investigating the dynamic properties of various constructions:

$$
\mathrm{H}(\omega)=\mathrm{X}(\omega) / \mathrm{F}(\omega)
$$

The relation between the values of the output $X(\omega)$ and input signal $F(\omega)$ determines the system transfer function - the impulse response function $H(\omega)$. The structure type, the research objectives and the required degree of accuracy determine the choice of the type and pattern of excitation of a structure. For laboratory tests, which require a high resolution, a harmonic (sinusoidal) excitation is typically used by sequentially changing the frequency within the set interval and measuring the response at selected points of the structure. For testing the conditions that fall outside of the laboratory environment, a widespread impact test method is applied. The key advantage here is the limited number of data measuring points. This method, which refers to an EMA method, was used for the experimental evaluation of the dynamic properties of the helicopter blade model.

The reliability of the evaluation obtained through the EMA is conditioned upon the capability to control the system excitation. This aspect limits the sphere of the application of the test Modal Analysis methods by confining them just to the laboratory environment or conditions that are close to the laboratory environment, since, under natural conditions, it is impossible to fully exclude external effects. The presence of the uncontrollable component of effect $\xi(t)$ distorts the evaluation of dynamic properties $H^{\prime}(\omega)$, which is going to include an error $\Delta(\omega)$ :

$$
\mathrm{H}^{\prime}(\omega)=\mathrm{X}(\omega) /[\mathrm{F}(\omega)+\xi(\mathrm{t})]=\mathrm{H}(\omega)+\Delta(\omega) .
$$

Under natural conditions, for dynamically stressed structures that are under load or in motion, it is possible 
to minimize the error using the OMA. This method allows determining the modal properties of a structure using only the system output data that is the dynamic signals of the stressed structure. The system response in such a case is caused solely by the environmental effect and no test excitation affects the system. The OMA makes use of a number of key approaches for the evaluation of modal properties, which are distinguished by the methods of data arrangement and processing. In this research, the authors considered the use of one of the OMA approaches - the Enhanced Frequency Domain Distribution (EFDD) - for the evaluation of the condition of a section of an operating pipeline.

For the practical application of Modal Analysis methods it is necessary to identify the major natural modes; therefore, an experimental phase was preceded by mathematical modelling using the finite-element technique (FET).

\section{Modelling of investigated objects}

The mathematical modelling of natural modes was performed by using the FET on the basis of the NEiNastran platform in CAD 2011 SP1.0. Two tasks have to be solved when modelling of investigated objects: one was the study of the model with the parameters of a natural object in order to identify the major oscillation modes, the second task was to evaluate the degree of mode sensitivity to the changes in the mechanical properties. For the pipeline model, a local defect was modelled, which simulated a welding joint defect, while for the helicopter blade model a global defect, which imitated the reduction in rigidity, was selected.

\subsection{The pipeline model}

For modelling a pipe, triangular parabolic elements evenly spread over the whole surface of the model were used as finite elements (FE). The maximum size of an element was set at $12 \mathrm{~mm}$. The analysis of the normal modes and frequencies of oscillation was done in respect of the first 20 natural modes, the displacement being used as a parameter.

Table 1 presents the calculated parameters of the natural modes of a pipeline model in the ideal pipe column, while Figure 1 demonstrates their mode shapes. The analysis showed that the majority of the modes are paired, that is they "sit" on the closely spaced frequencies and give similar modes with a $90^{\circ}$ phase shift. The modes are paired because of the symmetry of the pipe model cross section and its sealing. The identification of the modes allowed determining the two major mode groups and the two isolated modes. The first group includes 4 pairs of bending modes (1ab, 2ab, 4ab, 6ab), as shown in Figure 1, under which the pipe behaviour corresponds to the bending oscillations of a beam.
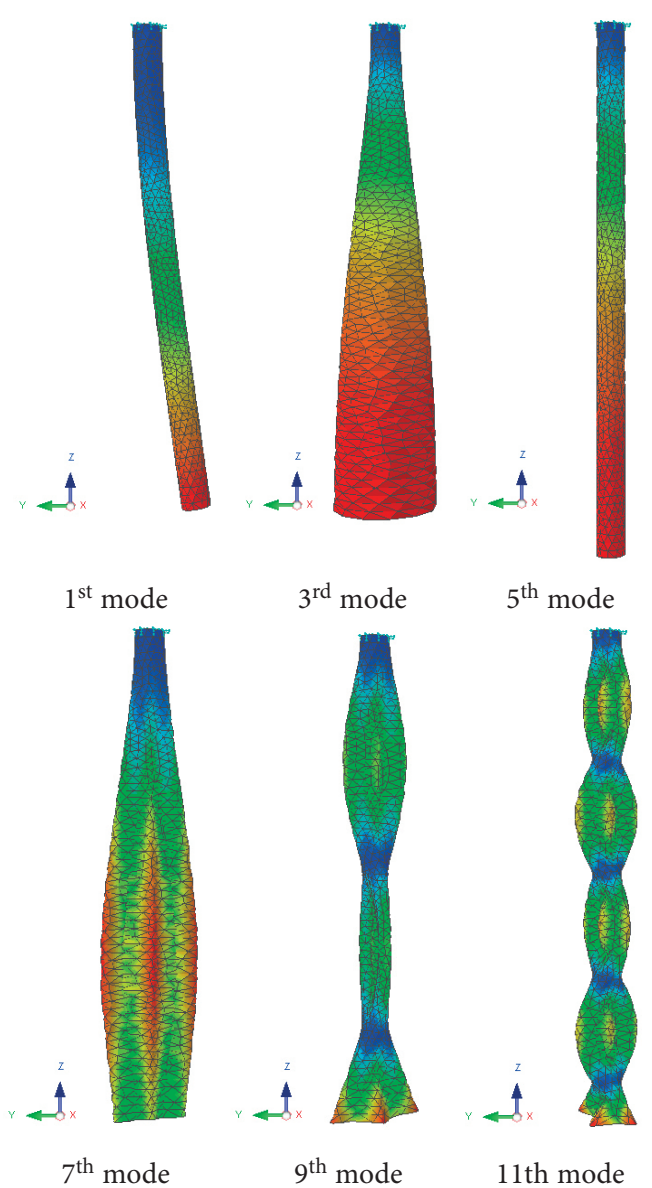

Fig. 1. Calculated modes.

Table 1. Comparison of modal frequencies between an "ideal" and "faulty" pipe

\begin{tabular}{|c|c|c|c|c|}
\hline \multirow[b]{2}{*}{$\begin{array}{c}\text { Mode } \\
\text { No }\end{array}$} & \multirow{2}{*}{ 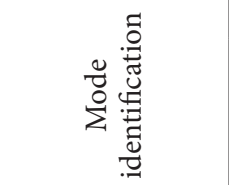 } & \multicolumn{2}{|c|}{ Frequency, $\mathrm{Hz}$} & \multirow{2}{*}{ 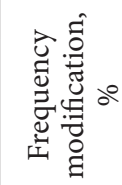 } \\
\hline & & 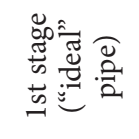 & 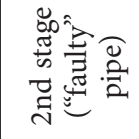 & \\
\hline $1 \mathrm{a}$ & \multirow{2}{*}{$1^{\text {st }}$ bending } & 36.19 & 35.76 & $1 \%$ \\
\hline $1 \mathrm{~b}$ & & 36.19 & 35.79 & $1 \%$ \\
\hline $2 \mathrm{a}$ & \multirow{2}{*}{$2^{\text {nd }}$ bending } & 219.35 & 212.61 & $3 \%$ \\
\hline $2 b$ & & 219.38 & 214.10 & $2 \%$ \\
\hline 3 & $1^{\text {st }}$ torsion & 487.61 & 478.06 & $2 \%$ \\
\hline $4 \mathrm{a}$ & \multirow{2}{*}{$3^{\text {rd }}$ bending } & 585.56 & 562.30 & $4 \%$ \\
\hline $4 \mathrm{~b}$ & & 585.67 & 567.75 & $3 \%$ \\
\hline 5 & $1^{\text {st }}$ longitudinal & 787.34 & 772.96 & $2 \%$ \\
\hline $6 a$ & \multirow{2}{*}{$4^{\text {th }}$ bending } & 1079.65 & 1063.49 & $2 \%$ \\
\hline $6 \mathrm{~b}$ & & 1079.84 & 1164.07 & $1 \%$ \\
\hline $7 \mathrm{a}$ & \multirow{2}{*}{$1^{\text {st }}$ membranous } & 1141.86 & 1128.12 & $1 \%$ \\
\hline $7 \mathrm{~b}$ & & 1141.99 & 1128.60 & $1 \%$ \\
\hline $8 \mathrm{a}$ & \multirow{2}{*}{$2^{\text {nd }}$ membranous } & 1149.15 & 1134.48 & $1 \%$ \\
\hline $8 \mathrm{~b}$ & & 1149.18 & 1134.59 & $1 \%$ \\
\hline $9 \mathrm{a}$ & \multirow{2}{*}{$3^{\text {rd }}$ membranous } & 1172.39 & 1159.15 & $1 \%$ \\
\hline $9 \mathrm{~b}$ & & 1172.45 & 1159.60 & $1 \%$ \\
\hline $10 \mathrm{a}$ & \multirow{2}{*}{$4^{\text {th }}$ membranous } & 1229.38 & 1215.44 & $1 \%$ \\
\hline $10 \mathrm{~b}$ & & 1229.45 & 1215.55 & $1 \%$ \\
\hline $11 \mathrm{a}$ & \multirow[t]{2}{*}{$5^{\text {th }}$ membranous } & 1338.21 & 1325.35 & $1 \%$ \\
\hline $11 \mathrm{~b}$ & & 1338.34 & 1326.02 & $1 \%$ \\
\hline
\end{tabular}


Under such deformations, the shape of the pipe model cross sections (circumference-shaped) almost does not change, only the longitudinal axis is bending. The two isolated modes are found within the same low-frequency range. The first one (3) is interpreted as the $1^{\text {st }}$ mode of torsion oscillations, in which the cross-sections of the pipe swivel about the longitudinal axis $Z$. The second one -the longitudinal mode (5) - is the one in which the free end of the pipe model shifts along the longitudinal axis $\mathrm{Z}$.

The second group includes 5 pairs of oscillation modes (7ab, 8ab, 9ab, 10ab, 11ab), where the wall of the pipe behaves as a shell. In each of the paired modes, the mode $\boldsymbol{b}$ is similar to mode $\boldsymbol{a}$, yet turned through $90^{\circ}$. At under membrane oscillations, the pipe model does not bend as a beam (the pipe axis is not deformed), which means there is no shift of the pipe axis; however, the cross sections get deformed, being "flattened" and "stretched" in perpendicular directions.

For the evaluation of the response (sensibility) of the oscillation modes to the local defect, a computational experiment was run, where a defect of a natural pipe was modelled with the help of the pipe model wall saw-through (less than $10 \%$ of the circumference), located circularly around the circumference.

The simplified analysis of the pipe model oscillations only suggested the numeric evaluation of the frequencies, while the evaluation of the mode shapes was limited to the visual assessment. However, since the frequency, which changes upon introduction of the defect, is one of the modal parameters, it can be assumed that the mode is changing along with the changes in frequency. The results of the computational experiment are shown in the column "faulty" pipe of Table 1. If we compare them with the data in the column "ideal" pipe, we observe that the pattern of changes in frequencies of different modes in response to the local defect differs. Thus, while the frequencies of the paired $1^{\text {st }}$ bending mode, which depends on the global properties of the modelled pipe, decreased by $1 \%$, the higher order mode frequencies that depend on the mass and rigidity distribution along the model length decreased to a greater extent (up to $4 \%$ ). For the membranous modes, the modal frequencies decreased to a lesser extent (1\%). The varying response of the modes to a local defect is illustrated by Figure 2 , showing the model behaviour along the $3^{\text {rd }}$ bending mode (4a, b in Table 1 ) in the two opposite oscillation phases. Evidently, the defect coincided with the maximum deformation spot for this mode; therefore, the parameters of that particular mode got distorted to the maximum extent.

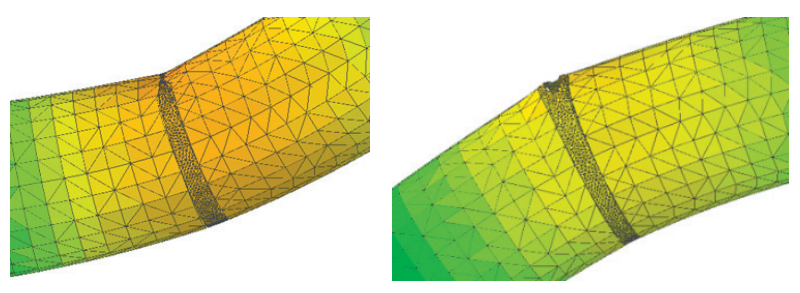

Fig. 2. Histogram of mode modification after introducing a defect model

\subsection{The blade model}

The lateral and longitudinal framing and skinning of the blade model is similar to that of a wing. The spar section of the blade represents an encasement formed by the back and front walls connected to each other by ribs forming the transverse framing and shaping the aerodynamic contour of the blade (Fig. 3a). The flexural rigidity is defined by the three longitudinal beams, which are rectangular in section and can be fixed to arbitrary positions. The correlation of the moments of inertia of the cross section of the beams in the vertical and horizontal position (orientation) is 10:1. By changing the beam orientation, it becomes possible to control the flexural rigidity of the blade model, in this way getting it modified along the full length. This type of modification simulates the loss if rigidity of the composite blades during operation, which primarily happens due to solar radiation.

Figure $3 \mathrm{~b}-\mathrm{d}$ demonstrates the oscillation modes for the first three modes shown as projections on the rotation plane and on the orthogonal plane. Table 2 shows the frequencies of the mentioned modes for the two positions of the beams: at the maximum rigidity towards thrust direction and the rotation plane. It is obvious, that the mode frequencies change in response to the changes of the rigidity of the structure.

Thus, the modelling demonstrated that the dynamic behaviour of the models is changing as a result of a global or local modification of the model parameters.

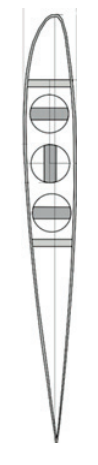

a)

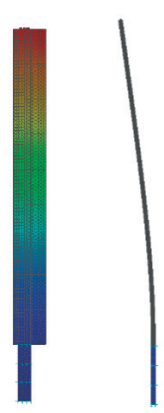

b)

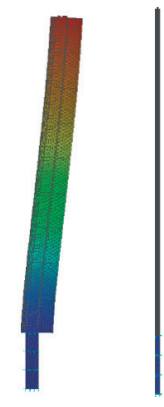

c)

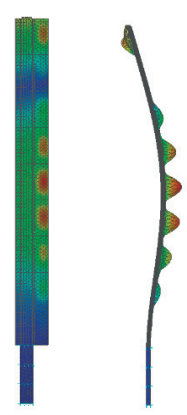

d)
Fig. 3. Blade model cross-section and mode shapes: a) model scheme, b) $1^{\text {st }}$ mode c) $2^{\text {nd }}$ mode d) $3^{\text {rd }}$ mode 
Table 2. Comparison of modal frequencies between blade models with a different stiffener orientation

\begin{tabular}{|l|l|l|l|l|}
\hline \multirow{2}{*}{$\begin{array}{c}\text { Mode } \\
\text { No }\end{array}$} & \multicolumn{4}{|c|}{ Frequency } \\
\cline { 2 - 5 } & $\begin{array}{c}\text { Stiffeners } \\
\text { in trust } \\
\text { direction }\end{array}$ & $\begin{array}{c}\text { Stiffeners } \\
\text { in rotation } \\
\text { pane }\end{array}$ & $\begin{array}{c}\text { Difference, } \\
\mathrm{Hz}\end{array}$ & $\begin{array}{c}\text { Difference } \\
\%\end{array}$ \\
\hline 1 & 42.49 & 42.26 & 0.23 & $1 \%$ \\
\hline 2 & 118.64 & 120.71 & -2.07 & $2 \%$ \\
\hline 3 & 249.53 & 237.00 & 12.53 & $5 \%$ \\
\hline
\end{tabular}

\section{Experimental investigation of the dynamic behaviour of the structures by applying EMA and OMA methods}

The experimental research, the results of which are presented here, was conducted using the multichannel equipment for conditioning and measuring signals from sensors distributed on the models and the software platform for dynamic signal analysis. The signals of the primary transducers, measuring dynamic parameters of the models, were used as the output data that characterize the condition of stressed structures. The result of data processing came as the parameters of the shapes, frequencies and damping factors of the natural modes of the structure, within the frequency range of 10-500 Hz.

\subsection{OMA of the experimental pipeline model}

The laboratory model of an operating pipeline was equipped with deformation sensors distributed along and circumferentially around the pipe. These sensors provide the data for the experimental determination of the dynamic characteristics of a laboratory pipeline model based on deformations measurement. The laboratory pipeline model has the form of a straight section of a hollow pipe. The pipe walls were excited by the impact of the liquid flow transported through the pipe. No test input of the pipe was applied during the measurement sessions. It was possible to adjust the degree of impact on the pipe walls by modulating the flow turbulence intensity. For that purpose, a controlled flow resistance in front of the pipe inlet was used.

Dynamic deformations of the pipe were recorded with the help of sensors - piezoelectric film transducers that transformed deformation into an electric charge. An ultra-light sensor attached to the pipe surface was exposed to stretching or compression together with the pipe wall, without affecting the mechanical properties of the pipe itself. At the same time, the dynamic range of the sensor is practically unlimited in terms of both the relative extension or compression and frequency. The time-variant charge generated at the output of the sensor during extension and compression is transformed into the alternate electric voltage by a preamplifier at the measuring channel input. The preamplifier is set on a flexible basis and, together with the sensor, represents a

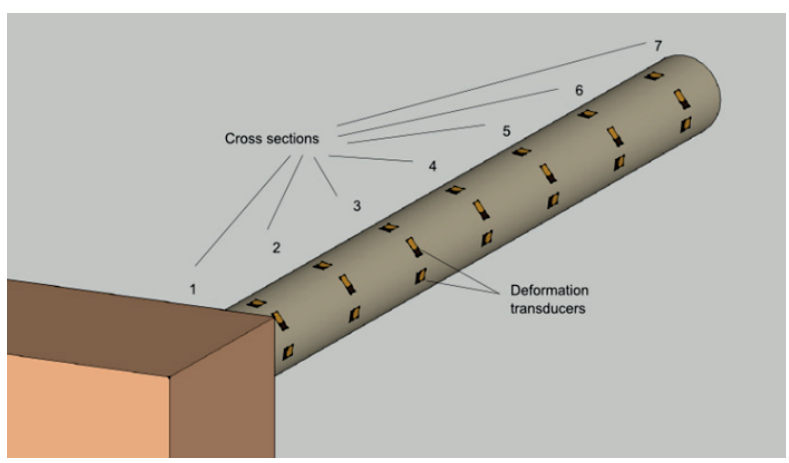

Fig. 4. Configuration of transducers on the pipeline segment

deformation transducer. 21 such transducers were set up on the tested segment of the experimental pipeline model in the seven evenly distributed cross sections (Fig. 4). In each cross section, the transducers were located along the intersection (generatrix) of the three planes going through the pipe axis. The planes were set at a $45^{\circ}$ angle against each other.

The transducers located on the top and side generatrix were set by its length along the pipe axis (longitudinal transducers), and their task was to measure the extension and compression of the "longitudinal" virtual fibres of the pipe. The transducers on the intermediary generatrix were directed around the circumference of the cross section and were supposed to sense cross-sectional deformation of the pipe wall (lateral transducers).

The input signals from all transducers are simultaneously sent via 21 channels to the data acquisition unit. The data from this unit is then transferred to the computer that saves the data and performs the OMA. The result of data processing is an eigenvector of the geometrical model of the pipeline model, which includes the FRF values for the description of each mode shape, as well as the mode frequencies, and the damping factors. The FRF values, given the assumed allowances, are proportional to the magnitudes of deformation at the corresponding points of the tested laboratory pipeline model.

The analysis of the oscillation mode shapes is performed with the help of diagrams. The deformation magnitudes are plotted by taking into account the phase (positive or negative) related to the cross section number. Depending on the location of the data points: - the top and side of the pipe (longitudinal transducers) or in between (lateral transducers), the magnitude values on the diagram are connected by a blue, red or green line (Fig. 5).

The modes were identified using their shapes mainly using an analytical method, based on the assumption that the pipeline model deformations were linked through the second derivate with the displacements that were calculated during modelling. The maximum displacement of the free end of the cantilever fitted pipe under the 1st oscillation mode is approaching zero deformation, which is observed in the 7 th section 

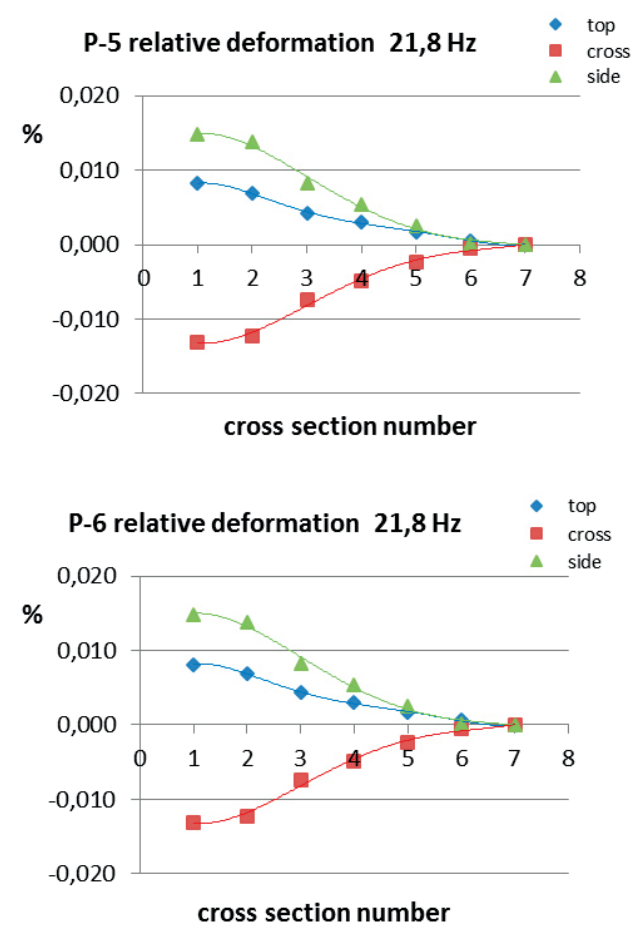

Fig. 5. Deformation diagrams of the 1st bending mode: (a) initial condition, b) faulted): top generatrix - blue rhombus; side - green cones; cross generatrix (lateral transducers) - red squads.

for all transducers. Contrarily, the minimum displacement in the area of the build-in support (1st section) corresponds to the maximum deformation of the pipe. A comparison of the deformation magnitudes along the top and the side generatrixes in the same cross section allows determining the oscillation phase in relation to the fixed coordination system of the laboratory facility. The magnitudes of the lateral sensors give an idea about the changes in the cross sectional shape of the pipe wall. Thus, the domination of the deformations of the side generatrix over the top generatrix (Fig. 5) is linked to a 620 slope of the pipe oscillation plane about the vertical line in the 1st bending mode. Negative values of the deformations of lateral transducers in the root section mean a "flattening"-type deformation in this cross section, which happens because the pipe bends near its closure.

The analysis of the experimentally obtained modal parameters demonstrated their similarity to the numerically estimated data, although the frequencies of the experimentally obtained modes have proven to be lower than estimated, due to the impossibility to implement the boundary conditions that are the same as those estimated during the experiment. Thus, the first bending oscillation mode, which, according to estimations, was supposed to be equal to $36 \mathrm{~Hz}$, in the laboratory facility, was just $21.8 \mathrm{~Hz}$. A comparison of the calculated and experimentally obtained oscillation modes demonstrated their close resemblance, proving the quality of the model. When analysing the properties through the OMA technique, no paired modes were revealed that would be characteristic to the digital model, since the pipe fixations of the experimental model did not correspond to the numerical one.

In order to investigate the diagnostic capabilities of the OMA technique, a local test defect was introduced into the laboratory pipeline model. The scale of the test defect was selected based on the task, which was to evaluate the lowest detection limit of the technique for the monitoring of the technical condition of the pipeline. The defect geometry corresponded to the above described model (section 3.1), the cut-through in the wall of the natural pipe was not complete - it was just $1 \mathrm{~mm}$ deep, or less than $30 \%$ of the wall thickness.

The analysis of the experimentally obtained modal parameters of the pipeline model demonstrated a variable response of different modes to the modification of the condition.

The deformation distribution diagrams obtained through the OMA and reflecting the modes of the laboratory pipeline model for the $1^{\text {st }}$ bending mode in good operating and defective conditions (Fig. 5), did not differ much from each other. The integrated difference of the FRF magnitudes under these two conditions did not exceed $0.2 \%$, while the frequencies differed by a mere $0.1 \%$, which corresponded to the estimated data.

At the same time, in several other oscillation modes, the differences proved to be more significant. For example, the difference of the FRF magnitudes for the $3^{\text {rd }}$ bending mode oriented in the inclined plane (Fig. 6) between the initial and defective condition was: in terms of magnitudes $-12.5 \%$, and in terms of frequency $-0.7 \%$. The modification of the mode shape occured primarily due to the change of the slope angle of the oscillation plane (in relation to the vertical plane) from $42^{\circ}$ to $25^{\circ}$ as a result of the defect.

The higher response of the $3^{\text {rd }}$ bending mode was conditioned by the fact that the location of the defect practically coincided with the position of the maximum deformation for this mode, which was already obvious at the modelling stage.

The results of the OMA application for the condition assessment of the experimental pipeline demonstrated a varying sensitivity of the modal parameters to defects. While the frequencies of the natural modes changed insignificantly, the modification of the pipe deformation shapes was quite obvious. This happened because the frequency of every mode is a global parameter of an object, while the deformation distribution (mode shapes) is the function of the local mass and stiffness distribution of the structure. Hence, the diagnostic efficiency of the modal shape parameters is much higher in comparison 
a)

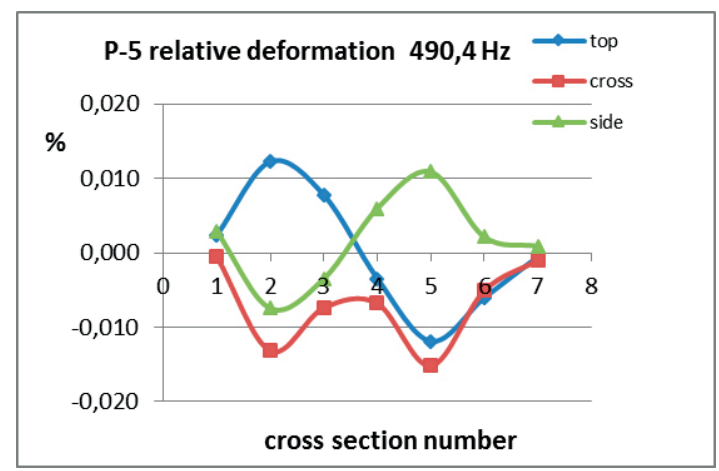

b)

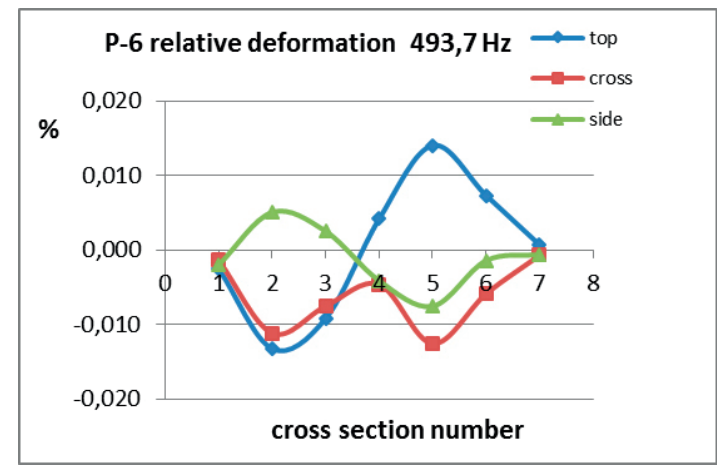

Fig. 6. Deformation diagrams of the $3^{\text {rd }}$ bending mode: (a) initial condition; (b) faulted: top generatrix - blue rhombus; side - green cones; cross generatrix (lateral transducers) - red squads.

to the mode frequency parameters. This explains the fact that the changes in the shape of the higher order modes, as a response to a defect, are able to become a reliable indicator of the alterations - even relatively smaller-scale local changes of the structural state.

\subsection{OMA of the experimental blade model}

Another technique of the modal analysis was applied for the experimental research on the dynamic properties of the three same-type helicopter blade models. The socalled "roving hammer" technique involves test impacts using a special hammer with a force transducer. By a sequence of impacts, the hammer "rounds" the blade model, while the response of each impact is measured by two triaxle transducers mounted on the blade tip and the root. The blade models are made of plywood and their construction is similar to the model described earlier (Fig. 7). By changing the position (orientation) of the beams, it becomes possible to control the bending rigidity of the blade, which is in this way modified along the whole length.

Three blade models were tested, each under two different conditions, when all the beams are set either vertically or horizontally. In the first case, the bending rigidity appears to be at the peak for the bending towards the thrust direction and at the lowest in the rotation plane. In the second case, it is the other way round.

A direct assessment of the change of the blade model stiffness at various blade positions was carried out a)

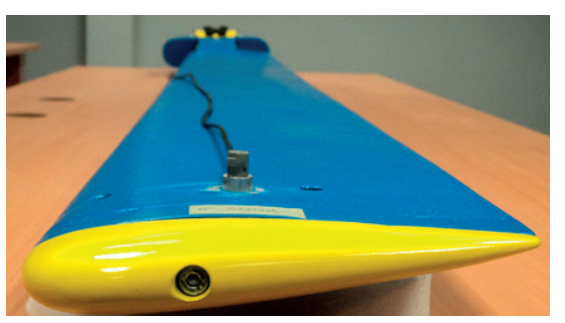

b)

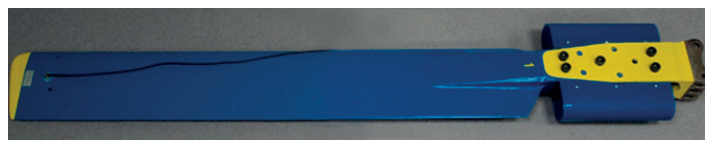

Fig. 7. Tested blade model: a) tip view, b) plan view

experimentally using the method of stepwise loading on the test bench. Simultaneously with the loading, the degree of the deflection of the control sections of the blade was measured. The difference in behaviour of the model parameters for both blade setting options is illustrated by the diagrams in Figure 8, as calculated for the blade No.1. It is obvious that, when all the three beams in each blade are set in a vertical position (simulation of the stiffness characteristic of a new natural blade), the bending of the model is almost twice as little (Fig. 8a) as the lowest stiffness configuration (Fig. 8b) which simulated an aged blade. A slight hysteresis at the loading and relief of the model is the result of the structural features of the blade model.

Table 3 ( $1^{\text {st }}$ row) demonstrates the results of the experimental assessment of the stiffness change of the three models that was caused by the stiffeners turn from minimum to maximum stiffness orientation.

a)

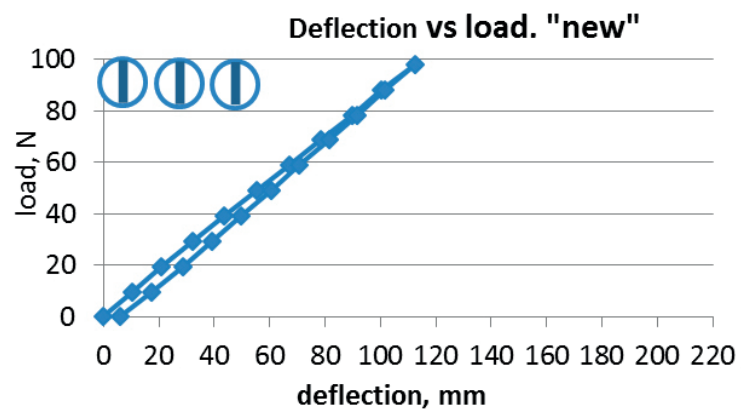

b)

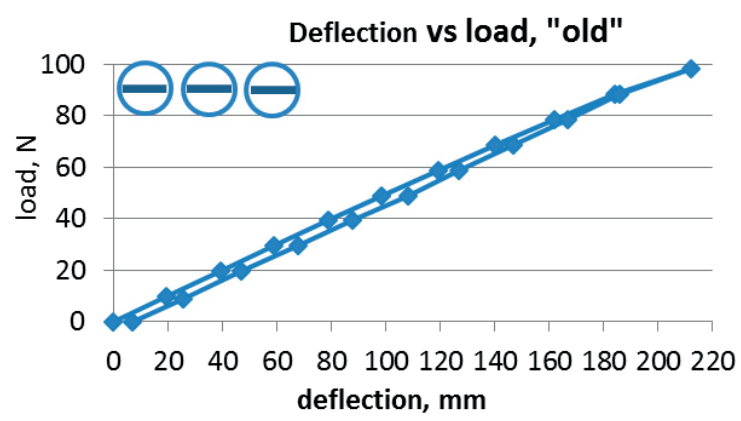

Fig. 8. Experimental assessment of the stiffness of the models: a) highest stiffness; b) lowest stiffness 
Table 3. Modal assessment (MA) of the blade model stiffness change (in \%) caused by stiffeners turn from minimum to maximum stiffness orientation.

\begin{tabular}{|l|l|l|l|l|l|}
\hline \multicolumn{2}{|l|}{} & \multicolumn{3}{|c|}{ Blade model No } & $\begin{array}{c}\text { Averaged } \\
\text { for all } \\
\text { blades }\end{array}$ \\
\hline No & Parameters & 1 & 2 & 3 & \\
\hline 1 & Experimentally measured & 79 & 93 & 79 & 87 \\
\hline 2 & $\begin{array}{l}\text { Exp. measured scatter } \\
\text { (to averaged) }\end{array}$ & -4.3 & 3.2 & 1.6 & \\
\hline 3 & $\begin{array}{l}\text { MA estimated by } \\
\text { frequency }\end{array}$ & 87 & 70 & 82 & 80 \\
\hline 4 & MA estimated by shapes & 69 & 25 & 82 & 92 \\
\hline 5 & $\begin{array}{l}\text { MA integrated } \\
\text { estimation }\end{array}$ & 78 & 95 & 82 & 85 \\
\hline 6 & $\begin{array}{l}\text { MA estimation error } \\
\text { (to averaged) }\end{array}$ & -3.8 & 5.4 & -1.6 & \\
\hline 7 & $\begin{array}{l}\text { MA estimation error } \\
\text { (to exp. measured) }\end{array}$ & -0.6 & 1.2 & -4.2 & -1.2 \\
\hline
\end{tabular}

Analysing the results of a direct measurement of stiffness, we notice that, for the $1^{\text {st }}$ model, it has increased to $79 \%$, when the beams were shifted from the horizontal position into the vertical. For the $2^{\text {nd }}$ model, it has increased to $93 \%$, and to $90 \%$ for the $3^{\text {rd }}$ model. The average change for all the three models was $87 \%$. The

a)

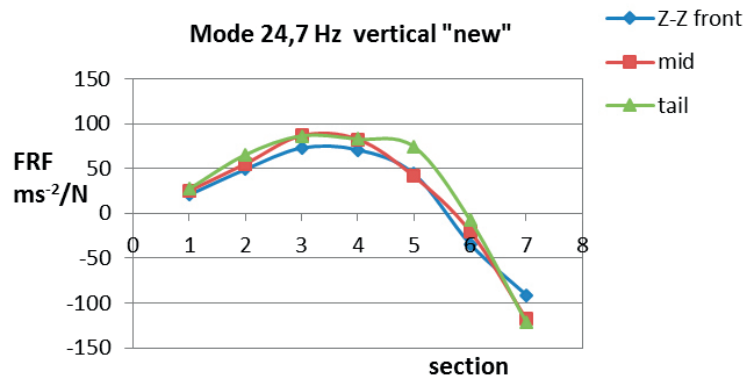

b)

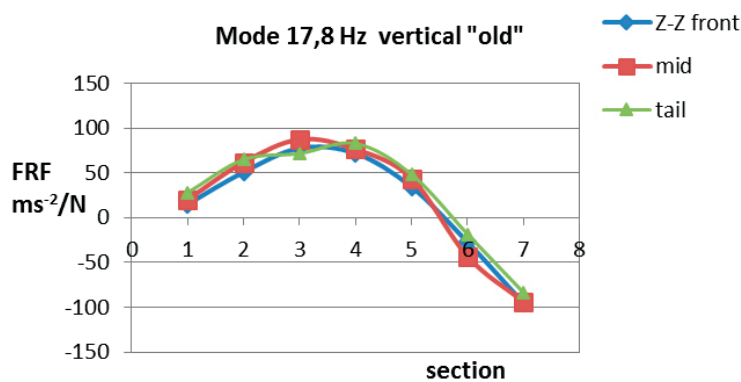

c)

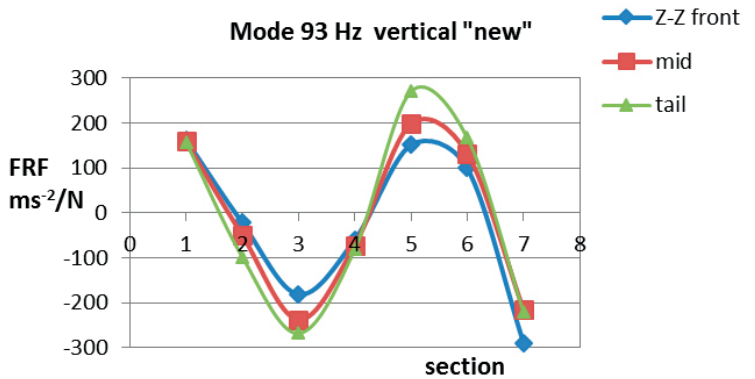

different response of the models to an identical change of the stiffness parameter is primarily the result of the difference in the mechanical properties of the models: the models were hand-made, not mass produced.

The study of the dynamic properties of the blade models included two simultaneous actions: excitation by using an impact hammer and response measurement. The excitation points on the blade surface were distributed in the seven cross-sections and three longitudinal axes, located along the leading edge, in the area of the spar and on the tail edge. The points of test impact by a special dynamic hammer were marked on the surface of the model. The FRF were determined sequentially at each point by multiple repetition and data validation.

The modal analysis of the experimentally obtained data of the models shows that there are 15 modes within the bandwidth of $10 \ldots 600 \mathrm{~Hz}$ which could be used for a subsequent comparative analysis in the two test conditions. The 6 identified modes were vertically bending, other 4 - horizontally bending, and 5 - twist modes. A comparison of the mode shapes is illustrated by the diagrams below (Fig. 9), where the deformation magnitudes were plotted by taking into account their phase (positive

d)

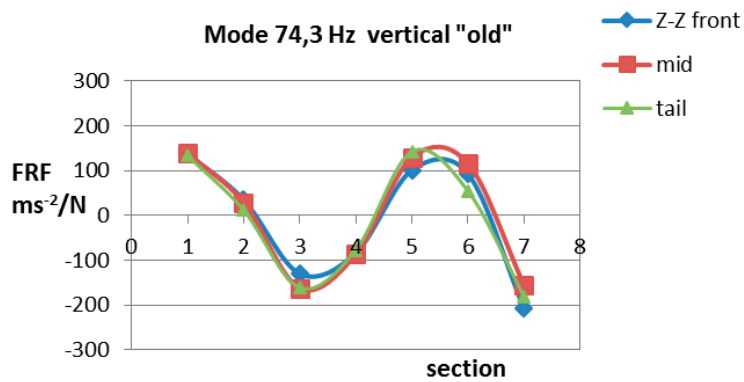

e)

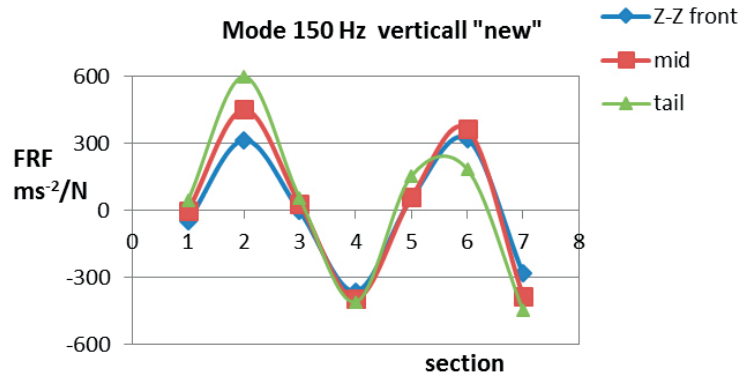

f)

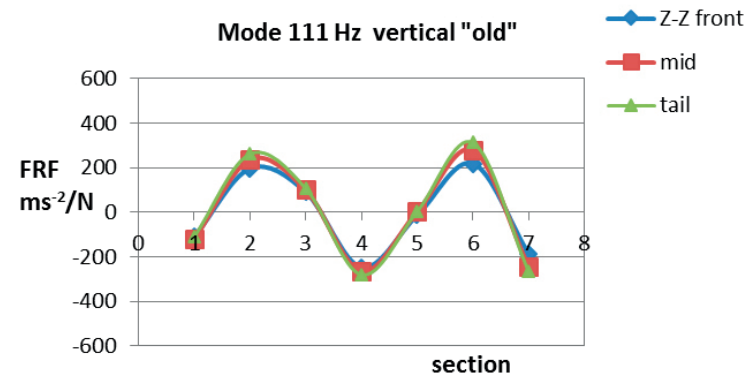

Fig. 9. Mode shape diagrams of the $1^{\text {st }}$ model for the $2^{\text {nd }}(a, b), 3^{\text {rd }}(c, d)$ and $4^{\text {th }}(e, f)$ bending modes in vertical direction (Y) under the conditions: a, c, e - "rigid-new"; b, d, f - "soft-aged". 
or negative), depending on the cross section number. In accordance with the position of the DOF on the front (leading) edge, the spar (mid) or the rear edge of the model, the values of the magnitudes on the diagram are connected by blue, red and green lines.

The frequency and shape of each mode were used as the parameters for comparison; additionally, the latter was determined by the magnitude with allowance for the phase. By way of example, the shapes of the three vertically bending modes are displayed in the diagrams in Figure 9. It is clearly seen that with the increase of the mode order, the magnitudes of the mode shapes decrease to a greater extent in response to the rigidity loss.

In assessing the relation between the changes of rigidity and the mode frequency, the following was taken into account: the mode frequency is proportional to the square root of the modal rigidity. Therefore, a reduction in frequency by, for example, $10 \%$ is supposed to correspond to a $19 \%$ decrease in the rigidity index. It was assumed that a linear dependence exists between rigidity and a deformation magnitude. Despite the errors allowed in the evaluation of the modal parameters the data scatter between blade models appeared to be quite small. Table 3 above shows the results of the comparison of the experimentally obtained evaluation of the changes in rigidity, based on the modification of modal parameters. Thus, in comparison to the reduction in the directly measured rigidity parameter of the 1 st model $1.79\left(1^{\text {st }}\right.$ row), the averaged (in terms of frequency and shape) measurement of the change of rigidity of this model proved to be almost the same -1.78 ( $5^{\text {th }}$ row of Table 3 ). It should be noted that, although separate measurements of the experimentally obtained parameters within an individual mode of, for example, the $2^{\text {nd }}$ blade model, may show certain discrepancy (in frequency 1.70, in shape 2.25), overall, the mode measurements (averaged) deviated from the average by as little as $5 \%$. It should also be noted that the scatter among the models is the result of the variation of their dynamic properties, because all models were handcrafted. At the same time, the variance among the results of direct measurement and the assessment results obtained via the modal analysis did not exceed $4.2 \%$.

Hence, the experimental verification of the modal analysis application proved that this technique is efficient for the estimation of technical condition, even given the absence of local defects. The fact that the integrated estimations for all the three models appeared to be pretty close to the experimentally measured parameters is conspicuous, which allows to assume that the given approach is suitable not only for the monitoring of the state of a certain structure during operation, but also for the diagnostics of same-type constructions.

\section{Conclusions}

The applicability of the experimental verification modal analysis technique for transport structures was analysed using functional laboratory models. Experimentally obtained natural modes were identified with the help of the finite-element modelling, which ensures the reliability of estimation of the changes in the modal parameters. The technique for determining the natural modes was refined by measuring the acceleration and deformation parameters. It was proved that the modal parameters obtained on the operating objects via the modal analysis techniques are able to serve as the diagnostic indicators of test defects of both local (in the pipeline model) and global (reduction of rigidity of the blade model) nature. It was revealed that it is more advantageous to use the mode shape parameters as the diagnostic indicators when compared to mode frequencies. Based on the comparison of the measurements of the model blade stiffness (rigidity) obtained during static tests and via the OMA, an assumption was made that the modal parameters obtained via the OMA can be used on operating structures for both structural monitoring as well as the diagnosis of the same-type constructions.

\section{Acknowledgements}

This work is written partly within research studies "Adaptive monitoring technologies for transport and energetics machinery rotating blades development" and „Perspective technology development of gas and fluid pipeline vibration monitoring" included in the project „Establishment of TRANSPORT MECHANICAL ENGINEERING COMPETENCE CENTER”, contract No. L-KC-11-0002 with Investment and Development Agency of Latvia and partly these results has been supported the funding from Latvia state research programme under grant agreement "Innovative and multifunctional composite materials for sustainable buildings - IMATEH”.

\section{References}

Allemang, R. J. 1999. Analytical and experimental modal analysis, UC-SDRLCN-20-263-663/664 1999. Cincinati: University of Cincinnati. 353 p.

Basseville, M.; Benveniste, A.; Goursat, M., et al. 2001. Output-only subspace-based structural identification: from theory to industrial testing practice, Journal of Dynamic Systems, Measurement and Control 123(4): 668-676. http://dx.doi.org/10.1115/1.1410919

Bendat, J. S.; Piersol, A. G. 1980. Engineering applications of correlation and spectral analysis. $1^{\text {st }}$ ed. New York: John Wiley and Sons, Inc. 302 p.

Dally, J. W.; Riley, W. F.; McConnell, K. G. 1984. Instrumentation for engineering measurements. $1^{\text {st }}$ ed. New York: John Wiley \& Sons, Inc.

Hall, S. R. 1999. The effective management and use of structural health data, in Proceedings of the 2nd International 
Workshop on Structural Health Monitoring, 8-10 September 1999, Stanford, CA, USA. 265-275 p.

Hall S. R.; Conquest T. J. 1999. The total data integrity initiative - structural health monitoring, the next generation, in Proceedings of the United States Air Force (USAF) Aircraft Structural Integrated Program (ASIP) Conference, 30 November - 2 December 1999, San Antonio, TX, USA. $2^{\text {nd }} \mathrm{ed}$.

Jolliffe, I. T. 1986. Principal component analysis. $1^{\text {st }}$ ed. New York: Springer-Verlag New York, Inc. 271 p. http://dx.doi.org/10.1007/978-1-4757-1904-8

Kessler, S. S.; Spearing, M. S.; Atalla, M. J., et al. 2002. Damage detection in composite materials using frequency response methods, Composites Part B: Engineering 33(1): 87-95. http://dx.doi.org/10.1016/S1359-8368(01)00050-6.

Kiddy, J.; Pines, D. 2001. Experimental validation of a damage detection technique for helicopter main rotor blades, Journal of System and Control Engineering 215: 209-221. http://dx.doi.org/10.1243/0959651011540996

Lisowski, W.; Uhl, T.; Malecki, J., et al. 2000. Investigation of structural dynamics properties of helicopter airframe, in Proceedings of the International Workshop on Noise and Vibration in Agricultural and Biological Engineering, 13-15 September 2000, Leuven, Belgium, 627-634.

Ljung, L. 1987. System identification: theory for the user. $1^{\text {st }}$ ed. Englewood Cliffs: Prentice-Hall, Inc. 519 p.

Magpantay, H. A. 2006. Modal identification from ambient vibration measurement: a technology for optimization of the performance of civil engineering structures. Naga: Ateneo de Naga University.

Tse, F. S.; Morse, I. E.; Hinkle, R. T. 1978. Mechanical vibrations: theory and applications. $2^{\text {nd }}$ ed. Englewood Cliffs: Prentice-Hall, Inc. 449 p.

Zhang, L.; Brincker, R.; Andersen, P. 2005. An overview of Operational Modal Analysis: major development and issues, in Proceedings of the $1^{\text {st }}$ International Operational Modal Analysis Conference, 26-27 April 2005, Copenhagen, Denmark. 